ISSN: $1130-3743$

\title{
BASES TEÓRICAS DE LA INTERVENCIÓN SOCIOEDUCATIVA DE LOS TÉCNICOS DE JUVENTUD
}

\author{
Theoretical bases of the socioeducational intervention \\ of youth technicians
}

\section{Bases théoriques de l'intervention socio-éducative des techniciens de jeunesse}

\author{
Myrte MOnSEny MarTíneZ* y Xavier ÚCAR MARTíneZ** \\ * Universitat de Girona. Facultat de Educación y Psicología. Departamento \\ de Pedagogía. Pl. Sant Domènec, 9. 17071 Girona. \\ Correo-e: myrte.monseny@udg.edu \\ ** Universitat Autònoma de Barcelona. Facultad de Ciencias de la Educación. \\ Departamento de Pedagogía sistemática y social. Edificio G6 Profesorado y Aulas. \\ Campus de la UAB. 08193 Bellaterra (Cerdanyola del Vallès). \\ Correo-e: Xavier.Ucar@uab.cat
}

Fecha de recepción: enero de 2012

Fecha de aceptación definitiva: abril de 2012

Biblid [(1130-3743) 24, 1-2012, 57-80]

RESUMEN

Este artículo tiene como finalidad ahondar en la dimensión educativa de las políticas de juventud así como en la tarea pedagógica que desarrollan los profesionales que las aplican: los técnicos de juventud. Es por ello que aquí se presentan el diseño y las conclusiones del reciente estudio desarrollado en el marco del programa de doctorado "Educación y Sociedad" de la UAB, en que se analiza la aplicación de estas políticas en las comarcas gerundenses durante la pasada legislatura, bajo el prisma innovador e integrador de un marco teórico que aúna diferentes perspectivas 
directamente vinculadas a las políticas juveniles y más concretamente a la definición que se hace de ellas en el Plan Nacional de Juventud de Cataluña. Estas perspectivas teóricas son: el enfoque biografista (Casal y otros, 2006); la teoría de las comunidades de práctica (Wenger, 2001); y la perspectiva del partenariado adultos-jóvenes, también denominada adultos como aliados (Checkoway, 1996). Dicho análisis es el que nos permite construir unas bases teórico-prácticas para la orientación de la intervención de los técnicos, ampliando sus referentes conceptuales, así como el reconocimiento de su tarea, aspirando a que una mejor intervención repercuta positivamente en los propios jóvenes.

Palabras clave: técnico de juventud, educación no formal, comunidades de práctica, pedagogía social.

\section{SUMMARY}

This article aims to deepen in the educational dimension of youth policies as well as in pedagogical professionals who develop it: the youth technicians. That is why we present the findings of recent study developed in the doctoral program "Education and Society" at the UAB, which analyzes the application of these policies in the Girona area during the last legislature, through the prism innovative and integrative theoretical framework that combines different perspectives directly linked to youth policy and more specifically to the definition that makes them in the National Youth Plan of Catalonia. These theoretical perspectives are: the biographical approach (Casal et al., 2006); the theory of communities of practice (Wenger, 2001); and the perspective of young-adult partnership, also known as adult as allies (Checkoway, 1996). This analysis is allowing us to build theoretical and practical bases for the guidance of the intervention of technicians, expanding their conceptual referents, and recognition of their work, aiming to improve the deployment a positive impact on young people themselves.

Key words: youth workers, non-formal education, communities of practice, social pedagogy.

\section{SOMMAIRE}

Cet article a pour finalité pénétrer dans la dimension éducative des politiques de jeunesse ainsi que dans le travail pédagogique que ses professionnels développent. C'est par cela qu'ici se présentent les conclusions de l'étude récemment développé dans le cadre du programme de doctorat "Éducation et Société" de l'UAB, dans laquelle est analysé l'application de ces politiques dans les comarques de Gérone pendant la législature passé, sous le prisme innovateur et intégrateur d'un cadre théorique qu'il ensemble les différentes perspectives directement liés aux politiques de jeunesse et plus concrètement -la définition qui est faite d'elles dans le Plan National de Jeunesse de la Catalogne. Cette analyse est celui qui nous permet de construire quelques bases théoriques-pratiques pour l'orientation de l'intervention des techniciens, en agrandissant ses référents conceptuels, ainsi que la reconnaissance de son 
travail, en aspirant à ce qu'une meilleure intervention répercute positivement sur les propres jeunes hommes.

Mots clés: techniciens de jeunesse, éducation non-formelle, communautés de pratique, pédagogie social.

\section{INTRODUCCIÓN}

Las primeras iniciativas en materia de intervención social dirigidas específicamente a la población juvenil nacen del impulso de la sociedad civil y no de las administraciones públicas o de la academia. Principalmente han sido las instituciones religiosas, las entidades sociales y las estructuras familiares y gremiales las primeras en atender las necesidades de este colectivo. No será hasta la configuración de las sociedades industriales y las formas sociales de urbanismo y modernidad que la administración pública comienza a mostrar interés por desarrollar sus propias propuestas en esta materia (Casal, 2002).

Este cambio de actitud no surge de forma casual sino que responde a modificaciones sociales y conceptuales de largo alcance. Un cambio que supone la dilatación del período vital de la juventud (Patón, 2003) como respuesta a unas necesidades de formación, especialización y demora de la incorporación de los jóvenes al mercado laboral.

La llegada de la democracia y el desarrollo de los ayuntamientos en la segunda mitad de la década de los 70 del pasado siglo supusieron un impulso en España de las políticas institucionales de juventud. Hay que apuntar, sin embargo, que los ajustes económicos y las elevadas tasas de desempleo juvenil existentes en aquellos momentos dificultaron el logro de los grandes fines que los discursos de aquellas políticas proponían. Según los primeros estudios sobre la evolución inicial de dichas políticas, se siguieron básicamente dos líneas de intervención: las centradas en las actividades de ocio y tiempo libre y las asociadas al compromiso de dar respuestas a las necesidades creadas por la crisis social y económica de los años 70 (Muñoz, 2002).

Como pone de relieve la cronología en el tema del trabajo socioeducativo con jóvenes, la urgencia por dar respuesta a las necesidades sociales anticipó la aparición de prácticas concretas a la creación de un discurso institucional o científico sobre las mismas. Esto no evita, sin embargo, la necesidad de proporcionar un marco legal y teórico a aquellas iniciativas que, por su relevancia e interés, deban ser reconocidas y ubicadas en alguna rama del conocimiento científico. A pesar de no ser ésta una necesidad intrínseca de la propia praxis, sí que es cierto que la ubicación de la misma dentro de alguna de las disciplinas científicas existentes nos ayuda a reconocer la racionalidad que opera en ella a la vez que nos facilita la tarea de darle consistencia, tanto a nivel práctico como teórico (Trilla, 1988). 
Esta coyuntura histórica, con la que se encuentran los profesionales de las políticas de juventud, es común a la de otros profesionales surgidos del proceso de especialización y profesionalización en algunos de los ámbitos de la educación no formal, como puedan ser, entre otros, los educadores sociales o los animadores socioculturales. A pesar de contar con un amplio bagaje en lo que a práctica profesional se refiere, estas profesiones presentan una trayectoria corta en lo que se refiere a su reconocimiento oficial, hecho que los sitúa en una relativa situación de precariedad en relación a otras prácticas profesionales del ámbito social.

Se genera al respecto una doble demanda por parte de estos colectivos. Por un lado, la necesidad de disponer de un marco legal que los ampare y del reconocimiento institucional que, desde su perspectiva, les corresponde. Por otro, la creación o sistematización de un conocimiento y unos saberes conceptuales que los orienten y prestigien. Dicho conocimiento ha de estar recogido en una formación específica o, mejor aun, en una titulación de nivel universitario. Ambos requisitos son esenciales en todo proceso de profesionalización de una función social, pues se requiere tanto de unas referencias y de un saber institucionalizado como especializado (Jolonch, 2002).

Es en este contexto de búsqueda de afirmación, reconocimiento y creación de un marco conceptual propio en el que enmarcar las funciones del técnico de juventud, donde se va a ubicar este trabajo. Más allá de aportar una propuesta de marco teórico y de analizar las políticas juveniles locales, nuestra investigación aspira a fundamentar la, desde nuestro punto de vista, clara finalidad educativa que las orienta (o habría de orientar).

\section{LAS POLÍTICAS DE JUVENTUD COMO INTERVENCIÓN SOCIOEDUCATIVA}

Las políticas juveniles tienen un claro antecesor en movimientos sociales como el movimiento "Scout", el asociacionismo o las corrientes de renovación pedagógica. Todos ellos han acumulado un amplio bagaje de técnicas y prácticas en la dinamización de actividades lúdicas o deportivas. En las últimas décadas las políticas de juventud han ampliado su concepción incluyendo otros aspectos que afectan a la vida de los jóvenes, como la emancipación o la participación social.

En el caso de Cataluña se puede ver este planteamiento reflejado en la caracterización de las políticas de juventud en el Plan Nacional de Juventud de Cataluña. Según este documento,

las políticas de juventud son políticas de definición y construcción de ciudadanía, políticas a través de las que se proporcionan elementos que permiten a las personas, como ciudadanos y ciudadanas, elaborar su propio proyecto de vida y participar en la construcción de proyectos colectivos (PNJCAT ${ }^{1}$, 2007, 18).

1. Pla Nacional de Joventut de Catalunya (2002), Barcelona, Generalitat de Catalunya. 
Un objetivo de estas políticas es facilitar el desarrollo de los jóvenes tanto en el plano personal como en el colectivo, manifestando con ello una voluntad que, sin ser exclusivamente educativa, sí que tiene un claro componente pedagógico.

Esta voluntad adquiere relevancia en tanto que el contexto sociohistórico y socioeconómico que les ha tocado vivir a los jóvenes de hoy en día favorece las "passioni tristi" (Contini, 2010), como resultado de la incertidumbre, inestabilidad y precariedad reinante. Estas pasiones tristes atraviesan y condicionan a estas generaciones en su vida cotidiana, en su modo de tomar decisiones y elecciones, en el modo como aprenden a gestionar sus emociones. Forma parte de una apuesta ética por la pedagogía no dejar únicamente en manos de la educación informal (publicidad, televisión o diarios) sus referentes sociales, culturales y simbólicos. El técnico de juventud es un agente educativo más que, con claras finalidades pedagógicas y en coordinación con el resto del entorno educativo de los jóvenes -familia e instituciones educativas-, tiene el encargo de la administración de facilitar inputs positivos que faciliten su emancipación, empoderamiento y ejercicio de la ciudadanía activa.

A pesar de que la dimensión educativa no es la única y principal impulsora de las políticas juveniles -entre otros motivos porque ya existen las políticas específicamente educativas- sí que se aspira a que todas las políticas desarrolladas desde este ámbito trabajen sus objetivos educativamente. Es por ello que a menudo se utiliza la animación sociocultural como recurso metodológico, puesto que, aunque el objetivo siempre sea la realización de un proyecto socioeducativo que puede tener temáticas diversas, la manera de trabajarlo pone a los jóvenes en el centro del proceso, estimulando y facilitando su autonomía y la vivencia creativa y responsable de su ciudadanía.

En la línea de la interpretación de la educación activa de Cirigliano (Santos, 2010) la aportación de las políticas de juventud a la educación no trataría tanto dar "un cambio cuantitativo del conocimiento, sino de un cambio cualitativo de la conducta que nos faculte para la búsqueda y hallazgo de verdades". Si los jóvenes deben afrontar el reto de aprender a presentar sus preguntas y demandas del modo adecuado en los nuevos escenarios (García, 2004), más que facilitarles una mayor cantidad de información, lo que conviene es ayudarles a desarrollar las capacidades que les permitirán emanciparse y participar de la sociedad.

Esta caracterización de las políticas de juventud nos sitúa en el ámbito de la educación no formal, en que a través de los campos más dispares -vivienda, trabajo, salud, cultura, etc.- se contempla la adquisición de diversas habilidades y competencias. Al igual que sucede con la animación sociocultural, pese a considerarse ésta una práctica educativa, no se puede decir que lo sea de forma exclusiva, sino que permite trabajar educativamente la consecución de otros objetivos (Trilla, 1988). En el caso de las políticas de juventud éstos pueden ir desde la emancipación familiar a la autonomía personal, la consecución de un trabajo o la expresión cultural. La animación sociocultural, así como las políticas de juventud, buscan generar una serie de efectos determinados en aquellos a quien se dirige, a la vez 
que se sitúan preferentemente fuera del radio de intervención de la educación formal.

Esta concepción de las políticas juveniles se inscribe en un enfoque crítico de la educación, a partir del cual se aspira a una praxis educativa en la que no hay escisión entre el pensamiento y la acción. El sujeto cognoscente es un sujeto actuante, el cual aprende a través de su praxis y su reflexión sobre la misma, haciéndose responsable de la historia y de una visión del mundo a través de ella (Giroux y McLaren, 1998)².

Partiendo de esta premisa pretendemos construir un referente teórico para aquellos que implementan estas políticas.

\section{PREgUNTA Y OBJETIVOS DE INVESTIGACIÓN}

La doble finalidad de la investigación que se presenta es construir un marco teórico que ayude a definir la intervención de los técnicos de juventud -en el marco de las políticas de juventud- a la vez que se profundiza en su profesionalización. La hipótesis de partida es que, cuanto más precisa sea la definición de sus funciones y tareas, de acuerdo con las necesidades del territorio, mayores y mejores serán sus resultados.

Para dar respuesta a estos objetivos la pregunta de investigación que se plantea es «Cuáles han de ser las bases que han de regir las funciones y tareas de los técnicos de juventud del territorio, de acuerdo con la evolución de las políticas juveniles de los últimos años?». Para abordar esta cuestión se formulan los siguientes objetivos:

I. Definir las variables, a partir del marco teórico elaborado, que determinan las políticas de juventud en el territorio.

II. Conocer las relaciones existentes entre las variables en dicho territorio a partir de la comparativa de las políticas de juventud de las diferentes Administraciones Locales -Ayuntamientos y Consejos Comarcales-.

III. Estructurar las bases que han de definir las funciones y tareas de los técnicos de juventud a la luz de los resultados obtenidos y del marco teórico elaborado.

3. LA CREACIÓN DE UN MARCO TEÓRICO INTERDISCIPLINAR COMO BASE EL DISEÑO DE LAS POLÍTICAS DE JUVENTUD Y PARA LA ACCIÓN DEL TÉCNICO DE JUVENTUD

Afirma Contini (2010) que no hay ninguna disciplina que sea totalmente autosuficiente. Esto es especialmente cierto respecto a la pedagogía en tanto que

2. En SANTOS (2010, 66). 
ciencia normativa. Hecho que significa que se nutre de otros conocimientos como la psicología, la antropología, la filosofía o incluso la neurociencia, entre muchos otros. Aunque estas contribuciones sean bienvenidas por la riqueza y multiplicidad de matices que nos facilitan, no deben desplazar la especificidad de la unidad que da la función educativa a sus actuaciones. Ésta tiene un estatus ético y epistemológico que dota a sus normas de una intencionalidad clara y de unas metodologías propias a la hora de actuar.

Asimismo, la transversalidad que caracteriza las políticas de juventud puede dar pie a que diversas disciplinas del conocimiento científico aborden su estudio. Consideramos que son tres los factores estructurales que determinan su articulación y que compete analizar para determinar el marco teórico que las justifica y explica. El territorio, los agentes implicados y su intervención son los pilares a partir de los cuales se construyen estas políticas y el motivo por el cual se han considerado tres perspectivas teóricas. La sociología de la juventud como prisma a partir del cual atender a la relación del contexto físico y social donde se desarrollan dichas políticas. En particular se ha optado por el enfoque biografista (Casal y otros, 2006) como superación de posturas anteriores -adultocráticas o de defensa de la ruptura generacional- que han generando discursos de gran calado como la nueva condición juvenil, pero que no dan una visión holística del fenómeno. La teoría de las comunidades de práctica (Wenger, 2001), como teoría social del aprendizaje que analiza el papel de los diferentes agentes implicados en la práctica (en nuestro caso la de las políticas de juventud) así como su actuación en torno a la empresa valorada. Y, finalmente, las aportaciones del trabajo social, como fundamento que recoge el planteamiento de la intervención desde una relación horizontal, a través de la perspectiva del partenariado adultos-jóvenes, también denominada adultos como aliados (Checkoway, 1996). Hay que apuntar que, de acuerdo con la apuesta ética por unas políticas de juventud pedagógicas que vayan más allá de la lógica de servicio y que apuesten por el fomento del empoderamiento de los jóvenes, la teoría social del aprendizaje de Wenger se constituye como eje vertebrador del discurso, siendo la función educativa del técnico la principal orientación de su actividad.

\subsection{El enfoque biografista}

En el marco de la sociología juvenil escogemos el enfoque biografista como el más adecuado, por su consonancia y complementariedad con el resto de dimensiones implicadas en este marco conceptual, así como por ser el más próximo a la definición de las políticas de juventud expuesta, al incluir el papel de los jóvenes como miembros activos de la sociedad. Este enfoque conceptual, más allá de tener como idea clave la consideración de las trayectorias de los jóvenes y sus propias decisiones y elecciones como aspectos fundamentales de su desarrollo, destaca por tres criterios definitorios (Casal y otros, 2006): 
- La transición de los jóvenes a la vida adulta como una articulación compleja de procesos de formación, inserción profesional y emancipación familiar.

- La emancipación social como construcción de un proceso ubicado en un marco sociopolítico determinado que configura un sistema político de transición.

- Las políticas de juventud y la intervención de sus técnicos, como actuaciones ubicadas en el entramado de un sistema político sociohistórico y geopolítico concreto.

Criterios que nos permiten incorporar al marco teórico, tanto la ubicación espacio-temporal de las políticas de juventud, como el papel determinante que tienen los jóvenes, técnicos y políticos en las mismas, condicionando los diferentes itinerarios y trayectorias de emancipación de los destinatarios.

La noción de trayectoria y de trayectoria paradigmática aparecen también en la línea teórica de las denominadas comunidades de práctica (Wenger, 2001) como punto en común entre la aportación sociológica y la dimensión pedagógica. Con la diferencia de que, en el primer caso, la trayectoria es el resultado de la incorporación de diversos elementos estructurales de la sociedad -situación socioeconómica, contexto familiar, etc.-, y en el segundo, representa la suma de factores intrínsecos al proceso de aprendizaje que las personas van sumando al largo de su vida -experiencia de negociación de significado, de construcción de un repertorio compartido, de participación social, etc.-.

\subsection{La teoría de las comunidades de práctica (Wenger, 2001)}

Las aportaciones pedagógicas de la teoría de las comunidades de práctica son el eje central del marco teórico de esta investigación y su contenido será la clave para interpretar los resultados obtenidos. Es en la misma definición del Plan Nacional de juventud de Cataluña donde hallamos la razón de su centralidad, al considerarse estas políticas facilitadoras de estímulos que permitan a los jóvenes crear su propio proyecto de vida, así como su participación en proyectos colectivos (PNJCAT, 2007, 18). Esta intencionalidad nos remite directamente a la vertiente pedagógica de las políticas de juventud, pues de ellas se desprende la aspiración de ser catalizador de aquellos elementos educativos que ayudan a los ciudadanos y ciudadanas a ser autónomos y responsables. A pesar de que, como ya se ha indicado, éste no es su único objetivo, sí que es la constante que acompaña todas sus acciones, convirtiéndose en parte fundamental de su razón de ser.

Este carácter educativo de las políticas de juventud se enmarca dentro del radio de actuación de la Pedagogía social, al concentrar el grueso de su actuación al margen de las estructuras formales del sistema educativo -escuela, instituto o universidad- no de forma excluyente, pero sí diversificando su campo de intervención. Dichas políticas se proponen como integrales y a tal efecto buscan incidir 
en el conjunto de los aspectos que afectan a la vida de los jóvenes. Tradicionalmente, cultura, ocio e identidad han sido algunas de las dimensiones más trabajadas. A pesar de que en la actualidad se ha querido poner mayor énfasis en aspectos nucleares como la vivienda o el trabajo, no por ello ha dejado la Administración de intentar dar respuestas globales a las necesidades concretas de este colectivo. En este sentido, la propuesta teórica de Wenger nos ofrece un marco muy apropiado para analizar y trabajar la relación educativa entre los jóvenes y la Administración a través de la figura del técnico de juventud, al poner al descubierto los diferentes factores y dinámicas implicados en las comunidades de práctica en tanto que espacios privilegiados de creación de sentido, identidad y consecución de objetivos vitales.

Las comunidades de práctica emergen de forma casi imperceptible para aquellos que las integran, como espacios de relación entre personas que comparten un interés por aprender y desarrollar competencias alrededor de un área o dominio en particular. Los objetivos que llevan a los participantes a formar parte de dichas comunidades pueden ser de lo más dispares, pero todos tienen en común la participación en un proceso de aprendizaje que les lleva a compartir:

- un compromiso mutuo con el conjunto de participantes,

- una empresa conjunta que los aúna,

- y un repertorio de significados compartidos.

Estos tres elementos les hacen competentes en la materia y les permiten reconocerse unos a otros. Todos los individuos pertenecemos a una u otra comunidad de práctica, puesto que todas las personas somos seres sociales y este es un factor del mismo aprendizaje, pero cada uno puede pertenecer a distintas y simultáneas comunidades de práctica. Es nuestro paso por las distintas experiencias que tenemos en cada una de ellas lo que va forjando nuestra identidad, nuestros conocimientos y nuestras trayectorias vitales. Las comunidades de práctica son una parte integral de nuestra vida diaria. Son tan informales y omnipresentes que rara vez son un centro de interés explícito y por esas mismas razones nos resultan también muy familiares.

En esta investigación hemos considerado que, en general, las distintas Administraciones locales suelen generar comunidades de práctica ajenas, o a menudo alejadas, de las dinámicas cotidianas de los jóvenes. De la misma manera que éstos constituyen comunidades de práctica, a partir de sus propios intereses, a las que la Administración difícilmente puede acceder.

En el caso de la Administración local, en tanto que comunidad de práctica, podría considerarse que la empresa conjunta que comparten todos sus implicados es el desarrollo o la implantación de un programa de gobierno. En él todos aquellos que participan en mayor o menor medida de dicha administración, independientemente de cuál pueda ser el trabajo que desarrollan, crean una práctica para hacer lo que es necesario hacer. Es así como todas las personas implicadas comparten: 
- Un compromiso entre ellas: la pertenencia a la Administración local en cuestión con todo lo que esto conlleva.

- Una tarea conjunta: por ejemplo, acabar la legislatura y acceder a la siguiente.

- Y un repertorio compartido: vocabulario administrativo, prácticas jurídicas, etc.

Los jóvenes por su parte también desarrollan sus propias comunidades de práctica; algunas intergeneracionales y otras específicas de su franja de edad. En todo caso, es habitual que algunas de ellas sean características de estos colectivos juveniles y este hecho se reconoce rápidamente por la falta de competencia de los adultos para desenvolverse en ellas. La diversidad de empresas que las pueden caracterizar es amplia, pero todas comparten los mismos requisitos de todas las comunidades de práctica:

- El reconocimiento y compromiso de los participantes -como miembros del colectivo en cuestión-.

- La empresa compartida; ya sea la creación de un grupo musical, la pertenencia a una sección juvenil de un partido político o la configuración de un grupo de estudio para la superación de una asignatura o curso, entre muchas otras.

- Y los significados compartidos. Quizá éste sea uno de los rasgos más característicos de algunas de las comunidades de práctica de los jóvenes; el uso de vocabulario específico de nueva creación que suele ser desconocido para las personas adultas.

Estas diferencias entre unas y otras hacen que, a menudo, se produzcan desencuentros entre ambas comunidades: las administraciones y los jóvenes. Entendemos, a pesar de todo, que es necesario establecer puentes de comunicación. Por un lado por el propio deber de la Administración de ocuparse de todos los ciudadanos incluidos los jóvenes. También, por el propio interés político de visualizar entre sus potenciales electores su programa de gobierno. Y, por el otro, por la necesidad, a veces no suficientemente reconocida o consciente por parte de los jóvenes, de acceder a las posibilidades o recursos que la Administración puede ofrecerles o denegarles en la construcción de sus propias trayectorias e itinerarios, ya sean individuales como colectivos.

Cabe destacar que uno de los efectos que se está produciendo a nivel global y que afecta particularmente a los jóvenes es el proceso de asunción de las trayectorias personales como consecuencia directa de las propias opciones y actos, es decir, como éxitos o fracasos personales obviándose, a menudo, la dimensión estructural y social que los afecta (Giménez y Llompart, 2004). Este desajuste entre realidad individual como hecho social y realidad individual como hecho subjetivo provoca un fenómeno de individualización subjetiva del riesgo que conlleva que, a pesar de que los condicionantes macrosociales se mantengan con tanta capacidad 
estructurante sobre la vida de las personas como en épocas anteriores, éstos no sean percibidos como tales (Furlong y Cartmel, 1997).

La aproximación de la Administración local a los jóvenes, así como la participación social de aquéllos, puede contribuir a paliar los efectos de esta visión subjetiva y reducida de los condicionantes sociales que afectan a los procesos de emancipación y autonomía de los jóvenes. La visión de la teoría del aprendizaje de las comunidades de práctica puede ser muy útil, al ofrecernos numerosos elementos de análisis que nos permiten enriquecer, tanto las posibles experiencias asociadas a las trayectorias de los jóvenes, como los modos de llegar hasta ellos. Con esta finalidad, el marco teórico de la investigación que aquí se expone propone que sea la figura del técnico de juventud la que desempeñe la función de correduría entre comunidades. Dicha función sitúa a este profesional en la periferia de ambas comunidades, puesto que no puede hallarse en el núcleo de ninguna de ellas si no quiere verse expuesto al rechazo de la otra, y le otorga la misión de traspasar información y conocimientos, a la vez que tender puentes, entre ambos colectivos. Ésta no es una tarea nada fácil, pero resulta de vital importancia para el desarrollo de ambas comunidades. El técnico de juventud, como "corredor" entre comunidades, ha de formar parte de ellas y ello implica estar comprometido con los miembros de ambas comunidades y con sus empresas, a la vez que es competente en sus actividades y conoce el repertorio compartido que se utiliza en cada una de ellas.

Para que esto sea así, es necesario que el técnico sea experto en el manejo de los factores que intervienen en las comunidades de práctica y, también, en los equilibrios y dinámicas que se dan entre ellos. En este sentido conviene que conozca que las comunidades de práctica se estructuran en torno a cuatro ejes:

A) El significado. Elemento que pone de relieve la práctica como proceso a través del cual los participantes de una empresa hacen de su experiencia, tanto en la tarea que les ocupa como en las relaciones con los compañeros que los acompañan, una vivencia significativa.

El significado es un elemento clave en el desarrollo de las actuaciones, tanto para que éstas tengan valor para aquellos a quien van dirigidas, como para que impulse la implicación de los mismos en ellas.

El significado no es, a pesar de todo, un factor estable, sino que está en continua negociación. Al igual que las comunidades de práctica tienen una vida y han de ir adaptándose a los cambios que les exige el entorno, el significado que opera en el seno de ellas ha de ir adaptándose también a las contingencias. Así mismo la negociación de significado es lo que permite a los miembros de las comunidades, así como a aquellas personas nuevas que se vayan incorporando al colectivo, la introducción de cambios o innovaciones. Éstos pueden producirse tanto en los conceptos y actuaciones que estén en juego, como en la ubicación de las personas que componen la red social de la comunidad. 
Conviene distinguir que en la negociación de significado operan dos mecanismos. La participación, como sistema introductorio de nuevos conceptos, interpretaciones o relaciones, hecho que garantiza la flexibilidad de las comunidades de práctica, así como la innovación, renovación e implicación en la construcción de sentido colectivo. Y la cosificación como contrapunto, es decir, como elemento estabilizador que consolida sin paralizar, permitiendo que las comunidades de práctica evolucionen sin desintegrarse. La cosificación es el proceso por el cual se producen abstracciones, símbolos, relatos o términos que cristalizan una parte de la práctica y que permite que todos sus participantes tengan referentes comunes. La combinación de ambos mecanismos es imprescindible y es lo que permite la supervivencia y el mantenimiento de las comunidades.

B) La comunidad. A pesar de que no toda comunidad está descrita por una práctica, sí que hay un tipo especial de comunidades que son las comunidades de práctica. La diferencia reside en que a las primeras quizá no las une una misma empresa o un compromiso mutuo entre sus miembros o incluso no comparten unos mismos términos mientras que las segundas sí. Éste podría ser el caso de una comunidad de vecinos. Si únicamente comparten un espacio o unos vínculos coyunturales, se puede decir que forman una comunidad, pero en ningún caso constituyen una comunidad de práctica. Si por el contrario estos vecinos crean una experiencia de aprendizaje compartido a raíz de aunar esfuerzos en torno a alguna reivindicación vecinal, puede ser que lleguen a constituir una comunidad de práctica, siempre y cuando compartan una tarea que les haga comprometerse los unos con los otros y que haga conocedores a sus miembros de aquellos elementos significativos que están en juego (actas, registros, etc.). Es así como podemos ver que el factor comunidad actúa como elemento delimitador y descriptivo de una práctica como unidad, pues de reivindicaciones vecinales pueden existir de muchos tipos y alrededor de muchas cuestiones, pero los términos en que lo hace cada comunidad de práctica concreta son exclusivos.

Más allá de la finalidad de la empresa en cuestión, se crea una red de vínculos sociales que tiene un peso por ella misma, reconociéndose sus miembros entre sí por tener la competencia de desenvolverse en dicha comunidad. Para poder ser competentes no solamente hay que formar parte de la comunidad, sino que también se requiere el compromiso con la red social que la constituye, así como el conocimiento de los términos en que se desarrolla esa práctica. La conjunción de estos tres requisitos es lo que permite la emergencia de las comunidades de práctica, así como las historias de aprendizaje compartido que les están asociadas.

Es por este motivo que, en relación con el tema que nos ocupa, conviene que el técnico de juventud sea competente en todas aquellas comunidades de práctica que por motivos laborales requieran de su implicación, en lo que al caso se refiere, tanto la Administración local como comunidades de práctica constituidas por los jóvenes del territorio. La función del técnico de juventud será, en este caso, la de trasladar conocimientos y aprendizajes de la una a la otra y viceversa. 
C) La práctica es por tanto el punto de encuentro donde se estructuran significado y comunidad dando paso a la creación de aprendizajes compartidos. Es pues punto neurálgico de confluencia de los diferentes elementos que configuran el aprendizaje social. En función de cómo se combinen estos elementos las comunidades de práctica se caracterizarán de un modo u otro, y tendrán una vida más larga o más perecedera, todo dependerá de su capacidad de reinventarse o estabilizarse.

La caracterización y el ritmo de los cambios vienen determinados por la participación y cosificación que se da en la negociación de significados, ya que son estos mecanismos los que generan los encuentros, continuidades y discontinuidades de los miembros de las comunidades. Al igual que el grado de implicación y participación de las personas en las comunidades puede ir variando según su trayectoria personal, la práctica que se desarrolla en ellas se debe ir adaptando a estos cambios. El modo como se lleve a cabo esta adaptación es lo que determina el tipo concreto de comunidad. Las hay más rígidas y conservadoras y las hay más flexibles e inestables. El punto de equilibrio entre ambas tendencias es lo que las hace más o menos efectivas y es un aspecto que siempre está en juego en función de la negociación de significado entre sus miembros.

Se supone que el técnico de juventud, en su desarrollo de la función de correduría, domina el reconocimiento y análisis de los factores y negociaciones que se llevan a cabo en el seno de estas comunidades, pudiendo incidir en ellas para mayor beneficio de las mismas y del entendimiento entre ellas. De manera que si, por ejemplo, una comunidad de práctica configurada mayoritariamente por jóvenes presenta dificultades para permanecer estable, debe poder recibir el apoyo del técnico a través de la facilitación de recursos de cosificación que ayuden a estabilizar los referentes y los compromisos asumidos con la empresa y con los compañeros. En caso de que los obstáculos sean, por ejemplo, la falta de jóvenes que asuman el cambio generacional, el énfasis deberá ponerse en el mecanismo contrario, y el técnico deberá fomentar la apertura y flexibilización de la comunidad, a través de la participación de potenciales nuevos miembros.

Las comunidades de práctica que puedan surgir vinculadas a la Administración también pueden presentar dificultades semejantes, ya sea por falta de flexibilidad a la hora de incorporar las propuestas de los jóvenes a sus dinámicas, como también por un exceso de complejidad en las cosificaciones elaboradas a la hora de hacerlas comprensibles para aquellos a quienes se dirige. En cualquiera de estos casos el técnico de juventud puede jugar un papel clave en la comunicación y entendimiento entre estos dos colectivos y en la mejora de las posibilidades de éxito de sus respectivos proyectos.

Por este motivo, el técnico de juventud debe demostrar su capacidad de:

- establecer relaciones de mutualidad en la comunidad,

- de compromiso con la empresa de aquellas comunidades de práctica con las que está en contacto y con profundidad suficiente como para desarrollar alguna responsabilidad hacia las mismas, 
- de utilización del repertorio compartido entre los miembros de las comunidades de práctica para dedicarse a ellas. Es decir, ha de saber comprender y usar tanto la jerga juvenil, como el lenguaje de la Administración pública.

Todo ello ha de lograrse sin que el técnico de juventud ocupe un papel central en ninguna de las comunidades, puesto que la función de correduría requiere del desarrollo de su tarea en la periferia de dichas redes sociales. Ésta es un área fecunda para el cambio, así como para el traspaso de información y aprendizaje, pues permite el acercamiento a las comunidades de personas que no son miembros de pleno derecho. Ésta por tanto es una posición ambigua, que sitúa al técnico ante los riesgos de desarraigo así como de encasillamiento ante las comunidades. Dichos peligros deben verse salvados haciendo uso de sus capacidades de multiafiliación, gracias a las cuales puede mantener su ascendente ante ambas comunidades sin perder su estatus de relativa neutralidad.

Este posicionamiento requiere de continuos procesos de coordinación, traducción y alineación entre perspectivas, no sólo para saberse mantenerse a flote entre las dos aguas, sino para llegar a tener influencia suficiente para conseguir que ambas partes se aproximen.

D) La identidad es otra de las dimensiones que está en juego en la dinámica de las comunidades de práctica y es precisamente la que nos permite recuperar la vertiente individual del proceso de aprendizaje. Hablar de identidad en términos sociales no supone negar la individualidad, sino que implica concebirla como un cúmulo de experiencias que se han ido depositando en la persona a través de sus diferentes vivencias en las comunidades de práctica. Construirse una identidad supone pues negociar a nivel social los significados de dichas experiencias, yendo más allá de la construcción de una trayectoria, y llegando incluso a determinar la identidad de una persona al identificarse ésta con unas prácticas, compromisos e ideas diferentes a lo largo de su vida. Nuestra identidad es pues un reflejo, tanto de nuestras capacidades, como de nuestras incapacidades, a la hora de participar en las comunidades de práctica así como de la manera que tenemos de afiliarnos a ellas. Desde esta perspectiva, la identidad se configura a partir de:

- la experiencia negociada,

- la afiliación o no afiliación a determinadas comunidades de práctica,

- la trayectoria de aprendizaje,

- los nexos de multiafiliación,

- y la relación entre la dimensión local y global entre las estructuras más próximas y las más amplias a las cuales pertenecemos.

Es por este motivo por el que el técnico de juventud ha de saber labrarse una identidad que le permita moverse entre aquellas comunidades de práctica en las que está implicado, para poder ser competente en las mismas, así como para poder obtener resultados de su trabajo con ellas. 


\subsection{La perspectiva del trabajo comunitario con jóvenes}

La última aportación teórica que viene a sumarse a este marco conceptual es la perspectiva del trabajo comunitario, desarrollada desde las estrategias de Adults

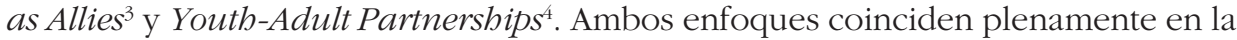
importancia del trabajo sobre las relaciones entre jóvenes y adultos como recurso estratégico para el logro, con éxito, del desarrollo de proyectos colectivos. Planteamientos que encajan a la perfección con la función de correduría que se defiende como referente a la hora de pensar el papel del técnico de juventud como agente educativo responsable del impulso e implantación de las políticas juveniles en el territorio. Se trata pues de dos perspectivas teóricas que complementan los dos primeros ejes que se han articulado en torno a la construcción de este marco teórico, la perspectiva sociológica de la teoría biografista y la perspectiva pedagógica de las comunidades de práctica.

Las estrategias de Adults as Allies y la de Youth-Adult Partnerships aportan a este marco conceptual una visión más pragmática de la actividad del técnico y de sus proyectos, ya que elaboran una teoría sobre aquellos aspectos prácticos del trabajo relativos a los vínculos entre jóvenes y adultos. En ella se detallan aspectos como:

- cuáles han de ser las condiciones de preparación de los miembros de cada una de las comunidades,

- a qué prejuicios habrá que enfrentarse,

- qué posibles obstáculos pueden surgir,

- qué aspectos hay que tener en cuenta en el proceso,

- y cuáles pueden ser los beneficios que se pueden obtener, entre otros...

Se distinguen tres tipos de desarrollo, el personal, que afecta a los participantes del proceso; el organizativo, relativo a la viabilidad y optimización de los proyectos conjuntos; y el comunitario, que se refiere a la creación de sinergias que permitan la mayor cohesión de la comunidad y el ejercicio de la ciudadanía de sus miembros.

3. Checkoway (1996) conceptualiza el vínculo de Adult as Allies, los adultos como aliados, como una alianza estratégica entre adultos y jóvenes en el desarrollo de proyectos comunitarios. Cómo propiciar este vínculo, cuáles son sus características y qué beneficios comporta son algunos de los aspectos que elabora en su obra.

4. CAMino (2000), seguidora y colaboradora de Checkoway, también ahonda en la relación entre adultos y jóvenes vinculada al desarrollo de proyectos colectivos y define la relación de Youth-Adult Partnerships, jóvenes y adultos como colaboradores, como un constructor multidimensional, en que participan principios y valores, así como habilidades y competencias, que permiten una dimensión estratégica de la acción colectiva. A partir de ahora, nos referiremos a esta relación estratégica como Y/AP. 
En el caso de la conceptualización Y/AP, el acento se coloca en otro aspecto de estas relaciones estratégicas, la multidimensionalidad, distinguiéndose tres vertientes:

- $\quad$ los principios y valores asociados a este proceso;

- las habilidades y competencias en juego y;

- la dimensión colectiva de dichas acciones.

Tanto los jóvenes como los adultos que participen de este tipo de iniciativas colectivas deben conocer que participan de este enfoque de trabajo comunitario, para ser conscientes de lo que supone su implicación en el mismo y no ser sus meros usuarios o beneficiarios. Si la aspiración del técnico de juventud es que jóvenes y adultos se hallen en la práctica del desarrollo de sus proyectos no se puede obviar la visualización de esta dinámica pues, de no ser así, difícilmente se conseguirá la implantación de principios tan básicos como el de mutualidad e igualdad entre las dos partes. Por ello se considera imprescindible la creación de un sistema de valores y creencias compartidos entre los implicados. Hecho que no conlleva la igualdad de pensamiento, pero sí unos valores y principios comunes que orienten la práctica. Posteriormente, para que todo el mundo pueda participar en condiciones similares, es imprescindible trabajar las habilidades y competencias de los participantes, para tratar de evitar al máximo los posibles obstáculos que pudieran surgir de la mala comunicación o incomprensión. Finalmente se aspira a que jóvenes y adultos se vean como socios en el desarrollo de las diferentes empresas de sus comunidades que, sin tener forzosamente objetivos compartidos, se benefician mutuamente de su interacción en el desarrollo de sus respectivos proyectos. Esta colaboración suele generar, de forma casi imperceptible, un mayor conocimiento de los unos respecto de los otros y aprendizajes compartidos.

Adults as Allies y $Y / A P$ son estrategias coincidentes en muchos aspectos, pero destacan sobre todo por la consideración de tres condiciones ineludibles en el proceso de relación entre jóvenes y adultos:

- la horizontalidad y equidad en las relaciones entre ambas partes;

- $\quad$ el reconocimiento y respeto de todos sus implicados; y

- la participación y responsabilización de todos ellos en el proyecto de entenderse.

Habiendo considerado desde la perspectiva sociológica la definición de las políticas de juventud, así como el papel que los jóvenes desarrollan en ellas; sumándole la dimensión pedagógica de las comunidades de práctica y la conceptualización de la función del técnico de juventud como una función de correduría; y finalizando con la consideración pragmática de la puesta en marcha de dichas relaciones a través de las estrategias Adults as Allies y Youth-Adult Partnerships; concluye la presentación del marco teórico que nos ha permitido llevar a cabo la investigación aquí expuesta. 


\section{CONCEPTUALIZACIÓN DE UN MÉTODO PARA EL ANÁLISIS DE LAS POlíTICAS DE JUVENTUD}

El siguiente reto se presenta a la hora de trasladar dicho planteamiento teórico a la dimensión práctica. Teniendo en cuenta que la postura conceptual de este estudio se articula alrededor del encuentro entre las tres perspectivas implicadas en la implantación de las políticas de juventud, la sistematización de dicho análisis ha de contemplar las variables implícitas en estas tres dimensiones. Es por ello que han sido tomados en consideración tanto los aspectos espacio-temporales en que se desarrollan dichas políticas, como los agentes sociales que intervienen, así como sus estrategias a la hora de implantar sus iniciativas en este terreno. De modo que podemos establecer un doble esquema de trabajo que puede ser observado en la Figura 1. Por un lado la representación gráfica del marco teórico a través de un diagrama de Venn que pone en relación las tres perspectivas que constituyen el posicionamiento de esta investigación. Y, por el otro, el paralelismo de dicho gráfico con la articulación de los tres descriptores que contienen las variables principales implicadas en dichas perspectivas.

El análisis de las características evolutivas de estos tres descriptores nos sitúa en el camino de dar respuesta a nuestra pregunta de investigación, al dibujar una fotografía de los resultados obtenidos durante la última legislatura que guíe las futuras actuaciones a partir de la contrastación de éstos con el marco teórico construido.

Es por ello que, en consideración a la perspectiva sociológica, se ha tenido en cuenta el territorio -representado por los municipios y las comarcas- como elemento clave de la dimensión espacio-temporal del objeto de estudio; pues es la ubicación sociohistórica y sociopolítica lo que nos permite atisbar los elementos estructurales que condicionan el desarrollo de la trayectoria de los jóvenes.

Jóvenes, técnicos y políticos debían aparecer irrenunciablemente en este proceso de sistematización de los componentes de la teoría de las comunidades de práctica, pues si estas comunidades son historias de aprendizaje compartido, está claro que ellos son los protagonistas de los proyectos construidos en torno a las políticas de juventud.

Finalmente, la dimensión más pragmática y estratégica de la puesta en práctica de estas políticas debía recogerse a través del estudio de los Planes Locales ${ }^{5}$ y Comarcales de Juventud $^{6}$, pues son éstos los instrumentos de planificación en que se articula este vínculo entre jóvenes y adultos alrededor de una iniciativa conjunta. En este sentido, a pesar de no llegar a un nivel de concreción como el del vínculo Y/AP o Adults as Allies, la tipología de los proyectos, así como su evolución, sí que nos pueden dar pistas de cómo se han ido construyendo estas relaciones hasta ahora.

5. Planes Locales de Juventud, a partir de ahora PLJ.

6. Planes Comarcales de Juventud, a partir de ahora PCJ. 
De este modo se colocan los tres ejes en torno a los cuales se estructura el sistema de análisis de esta investigación, al determinarse los descriptores que se corresponden con el marco teórico construido y que dan lugar a las variables que operativizan la recogida de datos.

Para llevar a cabo esta recogida de información, la presente investigación efectúa una revisión de las políticas juveniles llevadas a cabo en la demarcación de Gerona la pasada legislatura 2003-2007. Situando en 225 municipios y 8 consejos comarcales la población objeto de estudio. A partir de aquí lo que se lleva a cabo es un estudio descriptivo de desarrollo, que se concreta mediante la siguiente tabla comparativa.

\section{Figura 1. PARALELISMO ENTRE El POSICIONAMIENTO DEL MARCO TEÓRICO Y EL ANÁLISIS EMPÍRICO}

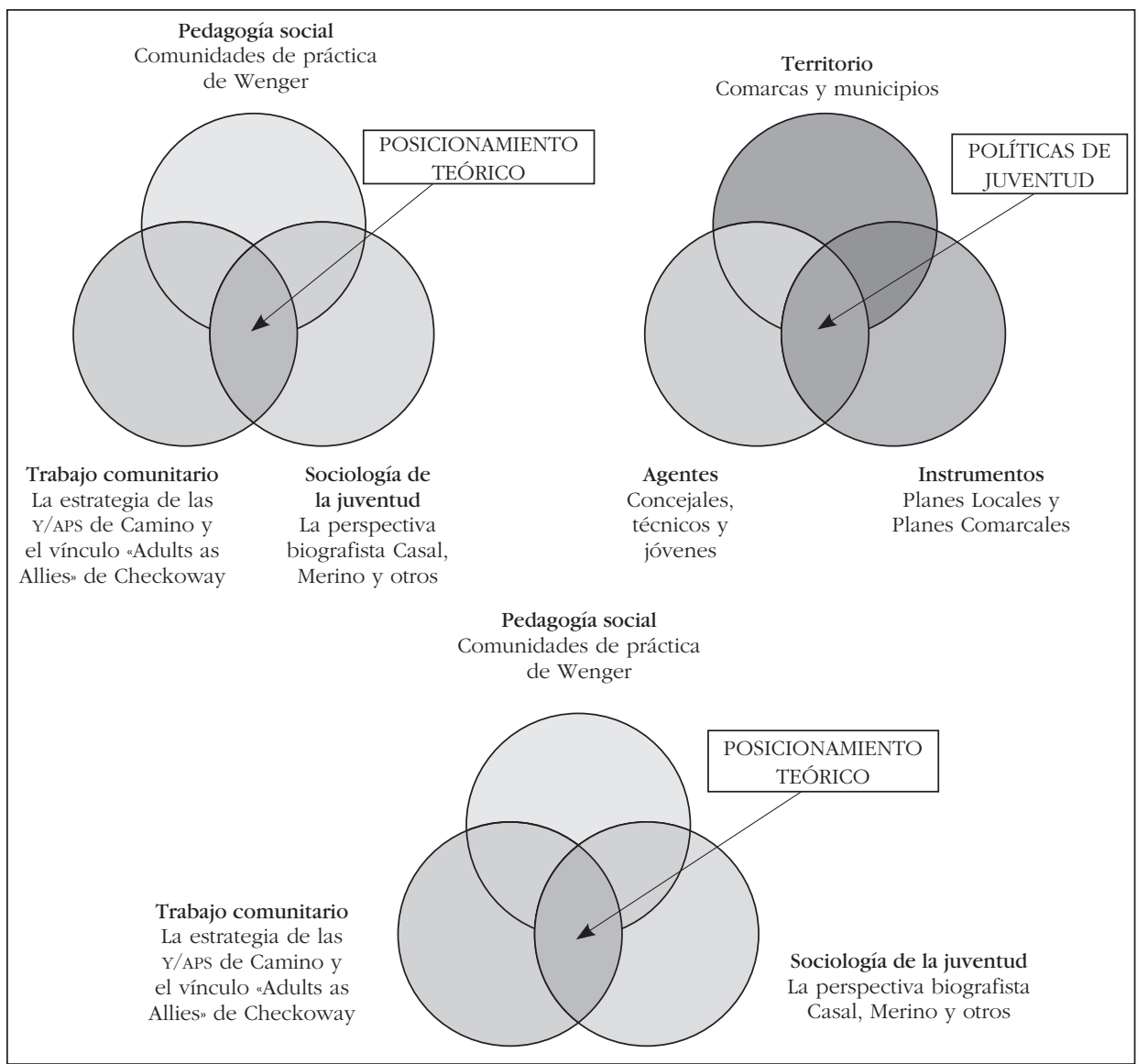


BASES TEÓRICAS DE LA INTERVENCIÓN SOCIOEDUCATIVA DE LOS TÉCNICOS DE JUVENTUD

\section{Figura 2. CuAdro DE ANÁlisis De los datos de la INVESTIGACión}

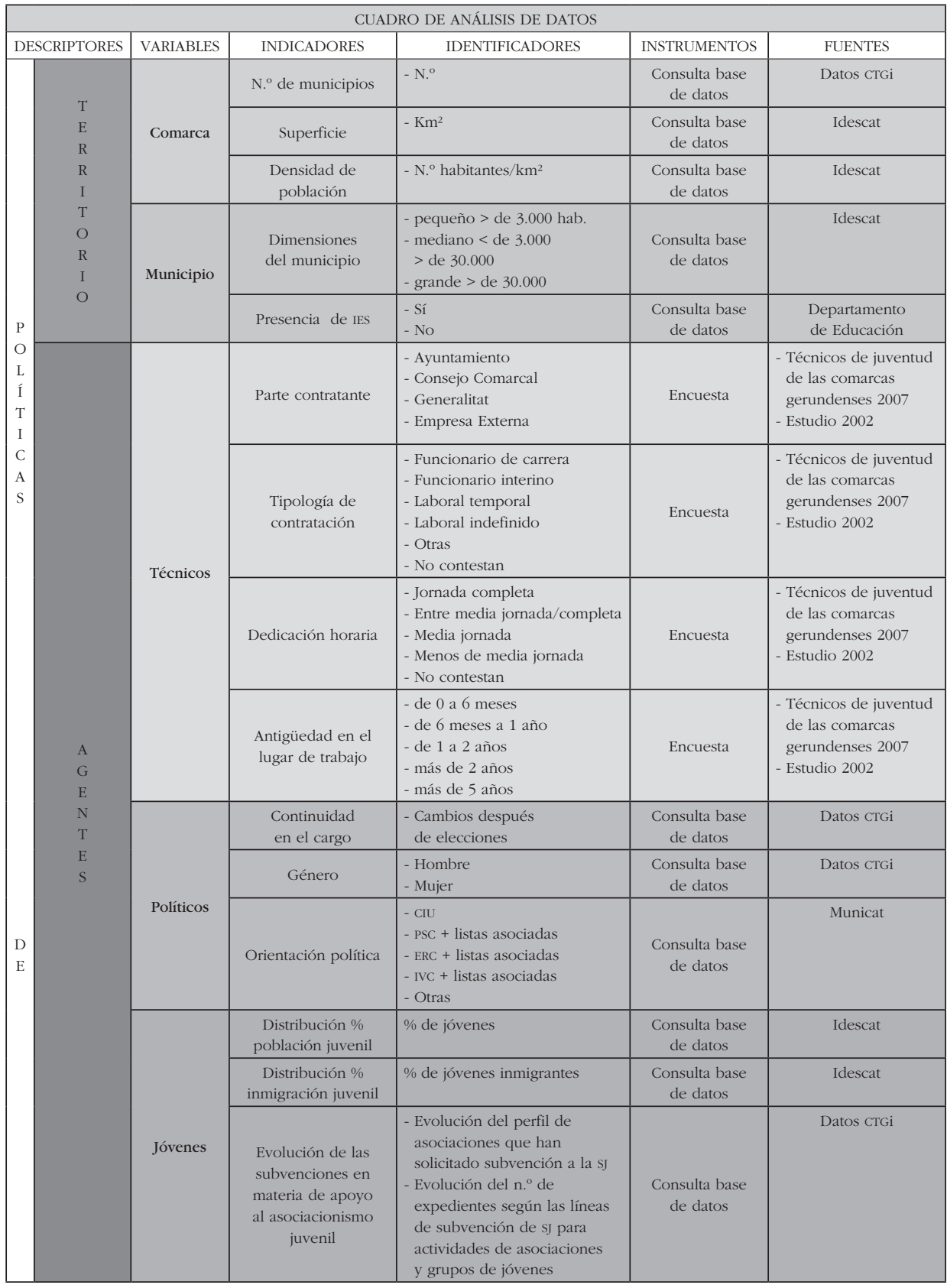




\begin{tabular}{|c|c|c|c|c|c|}
\hline \multicolumn{6}{|c|}{ CUADRO DE ANÁLISIS DE DATOS } \\
\hline DESCRIPTORES & VARIABLES & INDICADORES & IDENTIFICADORES & INSTRUMENTOS & FUENTES \\
\hline \multirow{4}{*}{$\begin{array}{l}\text { I } \\
\text { N } \\
\text { S } \\
\text { T } \\
\text { R } \\
\text { U } \\
\text { M } \\
\text { E } \\
\text { N } \\
\text { T } \\
\text { O } \\
\text { S }\end{array}$} & & $\begin{array}{l}\text { - Evolución del apoyo } \\
\text { a los proyectos } \\
\text { presentados por PCJ }\end{array}$ & $\begin{array}{l}\text { - Evolución del n. }{ }^{\circ} \text { de } \\
\text { proyectos y apoyo económico }\end{array}$ & $\begin{array}{l}\text { Base de datos } \\
\text { de CTGi }\end{array}$ & PCJ \\
\hline & $\mathrm{PCJ}^{*}$ & $\begin{array}{c}\text { Tipología de } \\
\text { proyectos } \\
\text { subvencionados } \\
\text { por PCJ }\end{array}$ & $\begin{array}{l}\text { - Mesa comarcal } \\
\text { - Estudios de comarca } \\
\text { - Difusión } \\
\text { - Técnico compartido } \\
\text { - Salud } \\
\text { - Participación } \\
\text { - Educación } \\
\text { - Laboral } \\
\text { - Vivienda } \\
\text { - Cohesión social } \\
\text { - Atención directa a los jóvenes } \\
\text { - Apoyo a PLj } \\
\text { - Otros }\end{array}$ & Consulta del PCJ & PCJ \\
\hline & & $\mathrm{N}^{\circ}{ }^{\circ}$ de PLI & $\mathrm{N}^{\circ}$ & $\begin{array}{c}\text { Base de datos } \\
\text { de cTGi } \\
\end{array}$ & Datos CTGi \\
\hline & $\mathrm{PLJ}^{*}$ & $\begin{array}{c}\text { Tipología de } \\
\text { proyectos asociados } \\
\text { a los PLj }\end{array}$ & $\begin{array}{l}\text { - Técnicos compartidos } \\
\text { - Participación } \\
\text { - Salud } \\
\text { - Laboral } \\
\text { - Educación } \\
\text { - Vivienda } \\
\text { - Dinamización } \\
\text { - Integración } \\
\text { - Batiburrillo } \\
\text { - Otros }\end{array}$ & Consulta del PLJ & PLJ \\
\hline
\end{tabular}

\section{CONClusiones}

Al final de este proceso los datos han revelado que, en el territorio que nos ocupa, el trabajo de proximidad no sólo es posible, sino que es casi la forma mayoritaria de trabajar, pues la gran mayoría de municipios ${ }^{7}$ no superan los 3.000 habitantes y en ellos el técnico de juventud puede desarrollar con los jóvenes un vínculo basado en la educación activa y en la promoción de su empoderamiento. En el caso de los municipios grandes ${ }^{8}$ esta manera de trabajar también sería viable si se dispusiera de más recursos, pero en la actualidad se vincula más a la prestación de servicios que no a una intervención continuada. A pesar de ello, el trabajo a partir de la perspectiva de las comunidades de práctica sería viable en ambos casos. En el primero con más facilidad, al ser el contacto entre el técnico de juventud y los jóvenes más cotidiano; pero también en el segundo, a través de un proceso más

7. Concretamente el porcentaje de los municipios menores de 3.000 habitantes en la demarcación de Girona según los datos del padrón de 2007 es del 81\%.

8. Los mayores de 30.000 habitantes representan el $2 \%$ y los medianos -entre 3.000 y 30.000representan el $17 \%$, 
largo de reconocimiento de las prácticas de interés del colectivo juvenil y la propiciación de la emergencia de dichas comunidades a partir del reconocimiento de los factores que facilitan la creación de las historias compartidas de aprendizaje.

Asimismo se ha constatado que el aumento de la presencia de estos profesionales y su progresiva estabilización ${ }^{9}$ ha ido a la par del incremento y mejora cualitativa de los instrumentos de aplicación de las políticas juveniles (PLj y PCJ), adaptándose cada vez más a las características y necesidades del territorio. Este hecho se refleja en las diferentes tipologías de proyectos, que en el caso de las comarcas y municipios grandes se caracterizan por su especialización, a diferencia de los municipios pequeños que destacan por sus líneas de trabajo más generalistas. En el primer caso el vínculo pedagógico que el técnico puede establecer con los jóvenes a través de las comunidades de práctica se centra en las prácticas asociadas a una determinada línea de trabajo, ya sea la dinamización del tiempo libre o la formación para la inserción laboral, entre otros ejemplos. En el caso de los municipios pequeños, las dimensiones del contexto geográfico permiten trabajar desde la diversidad de propuestas dirigidas a los jóvenes, tanto a aquellos que tienen comunidades más estables y "cosificadas" -asociaciones juveniles-, como a las emergentes -posibles grupos informales de jóvenes que puedan nacer alrededor de una práctica concreta-. En ambos casos el estudio de las comunidades de práctica nos facilita el análisis de las dinámicas que se producen en torno a la empresa valorada y nos ayuda a discernir cuáles son aquellos aspectos que se han de reforzar o flexibilizar (participación y cosificación en el sentido de Wenger).

Como refleja la teoría de las comunidades de práctica, la comunidad se construye alrededor de una práctica porque ésta tiene un significado para aquellos que la comparten; en consecuencia, el técnico no puede aspirar a propiciar o reconocer comunidades emergentes en propuestas desvinculadas de los intereses o necesidades de los jóvenes. El trabajo desde la proximidad implica el conocimiento de los centros de interés y de los posibles espacios de emergencia de comunidades de práctica que, dependiendo de los municipios o las comarcas, pueden estar

9. Los datos muestran la evolución de la consolidación de esta figura profesional. La mayoría de técnicos existentes en la actualidad están contratados de forma estable (funcionarios de carrera, 9\%; interinos, 3\%; o laborales indefinidos, 47\%) y aunque el aumento del porcentaje de técnicos contratados de forma temporal haya aumentado de un $14 \%$ a un $34 \%$, este hecho no es debido tanto a la precarización de este lugar de trabajo como a la incorporación de un mayor número de técnicos al conjunto del territorio. Se espera que éstos puedan paulatinamente consolidar su posición, para poder desarrollar un trabajo de calidad. Así mismo podemos percibir como la gran mayoría de ellos -69\%tienen una dedicación completa, a pesar de que se ve con una cierta preocupación el incremento de las jornadas inferiores -17\%-. Aun así, el hecho de que la mayoría de contrataciones de estos profesionales se concentre en los ayuntamientos y que sea precisamente en estas administraciones donde más ha aumentado la presencia los técnicos de juventud refuerza esta intervención desde la cercanía y desde la dimensión pedagógica. 
ubicados en un espacio u otro, pero que en todo momento buscan hacer de la relación entre jóvenes y adultos un espacio significativo.

Es cierto que la poca antigüedad de estos profesionales ${ }^{10}$ no nos sitúa en un escenario con mucha experiencia en este campo, pero sí que nos da la posibilidad de iniciar con cierta rapidez a estos técnicos en la aplicación de nuevas metodologías y planteamientos. Situación ésta que se repite en los políticos; cuya continuidad ha sido también muy baja. Este hecho a menudo conlleva la interrupción de las políticas o como mínimo la inestabilidad de las mismas. A pesar de ello, en lo que son las grandes líneas a nivel de orientación política y de perspectiva de género, no ha habido cambios substanciales.

Lo que se necesita ahora es que los técnicos tengan habilidad para resituarse en las nuevas comunidades de práctica que puedan surgir en la Administración a raíz de estos cambios políticos. De la misma manera, deberán ser diestros a la hora de transmitir y formar a sus nuevos referentes políticos en la realización de sus proyectos con jóvenes.

En lo que a los datos se refiere, el número de jóvenes que solicitan recursos a la Administración a través de subvenciones ha disminuido, y en especial aquellos que están asociados. Reflejo de este mismo hecho es que el único aumento que se ha producido en el número de solicitudes de subvención se haya dado en los colectivos de jóvenes no asociados. El técnico pues deberá trabajar con colectivos de jóvenes que acostumbran a ser poco constantes siendo éste un aspecto a abordar con las respectivas comunidades de práctica.

Asimismo, otra tendencia que deberá afrontar el técnico de juventud es la de la concentración de los jóvenes en los núcleos de población grandes, diversificando sus actuaciones en caso de trabajar en ciudades, y concentrando sus esfuerzos en llegar a los centros de interés en el caso de trabajar en los municipios pequeños a pesar de contar con menos servicios y recursos. En ambos caso, sin embargo, deberá poner atención en aquellas comunidades de práctica que puedan emerger entre los jóvenes como resultado del incremento del índice de inmigración juvenil, por lo que no sólo se deberá velar por su relación con la Administración pública, sino también por el propio vínculo con otras comunidades de práctica con las que puedan surgir afinidades.

Finalmente, por lo que respecta a los instrumentos utilizados hasta el momento en la implementación de las políticas de juventud -PLJ y PCJ- ya se ha destacado su evolución e incremento, pero también cabe mencionar su necesidad de estabilización. Hasta ahora la necesidad de desarrollo de dichas políticas ha sido imperiosa y han proliferado los proyectos de todo tipo. En la actualidad se impone la apuesta por priorizar aquellos proyectos que trabajen en profundidad algunas temáticas

10. El 41\% lleva menos de dos años desarrollando estas funciones; el 30\% lleva entre 2 y 5 años, y sólo el 29\% lleva más de 5 años. 
concretas de las políticas de juventud, en detrimento de los que tocan todas sus vertientes de modo superficial.

La planificación de múltiples y muy diversas actividades, sin conexión entre ellas, no posibilita el trabajo integral y menos aún la intervención desde la perspectiva de las comunidades de práctica. Un tal planteamiento corre el riesgo de tratar genéricamente todas las temáticas que afectan a la vida de los jóvenes, pero ninguna de forma significativa. Es esencial centrarse en aquellos aspectos que sean significativos y alrededor de los cuales se hayan constituido o se perciba que pueden emerger comunidades de práctica jóvenes. Sólo así se puede trabajar en aquellas condiciones que les han de permitir relacionarse con los adultos y crearse identidades de participación social y de ejercicio de la ciudadanía. Algo parecido sucede con los adultos y, más concretamente, con las comunidades de práctica que se puedan constituir alrededor de la Administración pública -sobre todo la local- pues sus miembros también han de aprender a desarrollar habilidades que les permitan interactuar con los jóvenes y atender sus necesidades. Ambos, adultos y jóvenes, cuando se hallan comprometidos con una comunidad de práctica, pueden establecer lo que Dennery $(1997)^{11}$ denomina "proceso de transferencia pedagógica", consistente en la capacidad de "transformar los conocimientos teóricos y prácticos en competencias clave, es decir, en saber, saber hacer y saber ser, y que se puedan poner en funcionamiento ante una situación profesional dada". El sujeto cognoscente es un sujeto actuante, una praxis en la cual asumimos la responsabilidad de la historia y de una visión del mundo que "aún no es" (Giroux y McLaren, 1998)12. Ésta es a nuestro entender la clave del éxito de las políticas juveniles y la que puede lograr en su desempeño no únicamente facilitar inputs educativos entre los jóvenes, sino también entre los adultos, propiciando la comprensión mutua y una mayor capacidad transformadora de la propia vida social y comunitaria.

A partir de aquí el trabajo de revisión de las políticas de juventud, al igual que la profundización en sus estrategias educativas de intervención, continúa, aspirando siempre a la mejora de las condiciones de vida de los jóvenes, así como a la contribución en su formación respecto del ejercicio de la autonomía y la ciudadanía. Se espera que la redacción de este estudio ayude en el reconocimiento de la figura de este profesional, a la vez que amplíe sus referentes teóricos, invitando a futuras réplicas o ampliaciones de este marco conceptual, pues la trayectoria teórica en este ámbito es aún parca y queda mucho camino por recorrer.

11. En Medina y SANZ $(2009,170)$.

12. En SANTOS $(2010,66)$. 


\section{REFERENCIAS BIBLIOGRÁFICAS}

Allerbeck, K. y Rosenmayr, L. (1979) Introducción a la Sociología de la Juventud. Buenos Aires, Kapelusz.

Camino, L. (2000) Youth-Adult Partnerships: Entering New Territory in Community Work and Research. Applied Developmental Science, 4 (1), 11-20.

Casal, J. (2002) TVA y políticas públicas sobre juventud. Revista de Estudios de Juventud, 59, $35-49$.

Casal, J.; García, M.; Merino, R. y Quesada, M. (2006) Aplicaciones teóricas y metodológicas a la sociología de la juventud desde la perspectiva de la transición. Papers, 79, 21-48.

Checkoway, B. (1996) Adults as Allies. Battle Creek, W. K. Kellogg Foundation.

ConTINI, M. (2010) Etica della professionalità educante, tra "passioni tristi", empowerment e resistenza. Teoría de la Educación, 22 (1) 19-41.

Diputació de Barcelona. Àrea d'Igualtat i Ciutadania, Oficina del Pla Jove (1999) Les polítiques afirmatives de joventut: una proposta per a la nova condició juvenil. Barcelona, Diputació de Barcelona y Ajuntament de Barcelona.

Dubet, F. (2002) El declive de la institución. Profesiones, sujetos e individuos en la modernidad. Barcelona, Gedisa.

Freire, P. (1983) Pedagogía del oprimido. México, Siglo XIX.

Furlong, A. y CARTMel, F. (1997) Young People and Social Change: Individualization and Risk in Late Modernity. Philadelphia, Open University Press.

García Canclini, N. (2004) Diferentes, desiguales y desconectados. Barcelona, Gedisa.

Generalitat de Catalunya. Departament de la Presidència. Secretaria General de JovenTUT (2003) Projecte Territori 2001-2003. Barcelona, Generalitat de Catalunya.

- PNJCAT. Pla Nacional de Joventut de Catalunya. Barcelona, Generalitat de Catalunya.

GIMÉNEZ, L. (2003) Las políticas de juventud: hacia unas políticas emancipatorias, en Aprendiendo a ser ciudadanos. Experiencias sociales y construcción de la ciudadanía entre los jóvenes. Madrid, INJuvE.

Jolonch, A. (2002) Educació y infància en risc. Barcelona, Pòrtic.

MEDINA, Ó. y SANZ, F. (2009) El reconocimiento y la acreditación de la experiencia. Teoría de la Educación, 21 (2), 165-193.

MuÑoz, L. B. (2002) Políticas integrales de juventud versus políticas afirmativas. Presupuestos para un debate. Revista de Estudios de Juventud, 59, 70-78.

PATón, J. M. (2003) Joves adults i polítiques de joventut a Europa. Marc conceptual, punts de partida $i$ criteris per a l'anàlisi transnacional comparada de les polítiques de joventut a Europa. Barcelona, Generalitat de Catalunya.

Planella, J.; Vilar, J.; Pie, A.; Sáez, J. y Úcar, X. (2006) La pedagogía social en la sociedad de la información. Barcelona, Editorial UOC.

Quintana, J. M. (1984) Pedagogía social. Madrid, Dykinson.

SAntos, M. (2010) Pedagogía, historia y alteridad. Teoría de la Educación, 22 (2), 63-84.

SCHLEGEL, A. y BARRY, H. (1991) Adolescence: An anthropological inquiry. New York, Free Press.

SOlER, P. (2002) Els tècnics en la política de joventut de l'Administració pública catalana. Barcelona, Generalitat de Catalunya.

TriLla, J. (1988) Animación sociocultural, Educación y Educación no formal. Educar, 13, 17-41.

ÚCAR, X. (2002) Medio siglo de animación sociocultural en España: balance y perspectivas. OEI-Revista Iberoamericana de Educación. Consultado el 20 de octubre de 2011. http:// www.rieoei.org/edu_soc1.htm.

Wenger, E. (2001) Comunidades de práctica. Aprendizaje significado e identidad. Barcelona, Paidós. 